\title{
The geomagnetic storm of August 2010 as a lesson of the space weather modeling
}

\author{
Miloš REVALLO ${ }^{1}$, Fridrich VALACH ${ }^{2}$, Magdaléna VÁCZYOVÁ ${ }^{2}$ \\ ${ }^{1}$ Geophysical Institute of the Slovak Academy of Sciences \\ Dúbravská cesta 9, 84528 Bratislava, Slovak Republic; e-mail: geofmire@savba.sk \\ ${ }^{2}$ Geomagnetic Observatory, Geophysical Institute of the Slovak Academy of Sciences \\ Komárňanská 108, 94701 Hurbanovo, Slovak Republic \\ e-mail: fridrich@geomag.sk; magdi@geomag.sk
}

\begin{abstract}
A model of the magnetic storm of August 4, 2010, is presented in the paper. Simulated Dst index series is obtained from an empirical model of the solar wind magnetosphere interaction and is compared with real data. In broader context, the underlying study can be conceived as an example of the space weather modeling. A brief survey of the approaches to this topic is provided in the paper. In particular, the link is made to the previous studies based on artificial neural networks which could be beneficial for the magnetic storm modeling as well.
\end{abstract}

Key words: space weather, magnetic storms, magnetosphere, Dst index

\section{Introduction}

The concept of space weather is used to describe the conditions in space that can affect both the Earth and its technological systems (Robinson and Behnke, 2001). It is a consequence of the behavior of the Sun and the interaction of the Earth's geomagnetic environment with the solar wind. Space weather research is a relatively new enterprise that combines space sciences with the development of practical applications for the benefit of human activities affected by the space environment. Adverse conditions in the near-Earth space can influence the performance and reliability of spaceborne and ground-based technological systems, and can endanger human life or health. Effects include the temporary and sometimes permanent failure of satellites, the degradation or disruption of communication and navigation, electric power distribution grids, and the exposure of astronauts to 
harmful doses of radiation. Ultimately, such behavior can lead to a variety of socioeconomic losses.

The near-Earth environment is influenced especially by the properties of the solar wind plasma and the frozen-in interplanetary magnetic field (IMF). A variety of physical phenomena are associated with space weather, including geomagnetic storms and substorms, energization of the van Allen radiation belts, ionospheric disturbances and scintillation, auroras and geomagnetically induced ground currents. Coronal mass ejections (CMEs) are the primary cause of the largest and most damaging space weather disturbances. They are the most energetic events in the solar system. CMEs are expulsions of large quantities of plasma and magnetic field from the Sun's corona (Kim et al., 2005). The occurrence rate of CMEs approximately follows that of the solar (sunspot) activity cycle. Strong ejections are accompanied with shock waves impacts on the Earth's magnetosphere resulting in geomagnetic storms. Let us mention the recent geomagnetic storm of August 4, 2010, believed as a possible onset of the new solar cycle.

It is now no longer enough simply to be able to understand and predict the weather conditions of the atmosphere. There is increasing demand to understand and predict the conditions in the near-Earth space as well. This need will grow even greater as society's dependence on space and spacebased technologies steadily increases over time.

The underlying study is an attempt towards the space weather modeling using a model of the solar wind magnetosphere interaction. In Section 2 a brief survey of approaches to the space weather modeling is provided. In Section 3 the mathematical model is described and in Section 4 the model output is compared with real satellite data. In Section 5 the main findings are summarized.

\section{The space weather modeling}

There is an effort to develop physics-based, high performance models of the Sun-Earth system which can operate in real time using data from solar observations and upstream solar wind measurements. Simulations of synthetic space weather events are possible by taking advantage of parallel computing and using sophisticated numerical methods together with advanced statistics. 


\subsection{Numerical approach}

Global magnetohydrodynamic (MHD) computational models based on first principles have been used to model the time-dependent interaction of the solar wind with the Earth's magnetosphere (Gombosi et al., 2001). Geomagnetic activity is usually triggered by magnetic reconnection processes at the nose part of the magnetopause. Intense long-duration southward IMF is believed to be one of the most important causes for a major magnetic storm, which can produce severe space weather. Magnetic storms occur when the number and energy of charged particles in the outer radiation belts increase significantly. The penetration of particles is a consequence of a southward turning of the IMF. Thus for space weather applications we are most interested in the solar wind velocity, the mass density, the temperature, and the strength and orientation of the IMF. These quantities can be used as an input information for global models.

Besides the global modeling, there is an alternative approach of empirical (quantitative) modeling (Romashets, 2008). The use of empirical models for the purposes of forecasting has the advantage of being less computationally demanding than the MHD models. Quantitative models should be able to replicate essential features of the response of the magnetospheric configuration to the variable external input. It is important to develop objective assessment criteria to determine which empirical models are most accurate, and where weaknesses in the models exist. Forecasting geomagnetic activity depends critically upon our ability to forecast the properties of the solar wind and its imbedded magnetic field, particularly the north-south component of the IMF when it encounters the magnetopause.

\subsection{Statistical approach}

The method of artificial neural networks (ANN) as a method of advanced statistics represents a powerfull tool when applied to space weather. The modeling capability of the ANN is in its ability to learn the mathematical function underlying the system operation. If the network is designed and trained properly, it can perform generalisation rather than simple curve fitting. This approach is especially useful when the information about physical system may be incomplete. The ANN possesses an input which can be fed with the data on solar activity. For a given data set, the ANN can 
be trained to provide the geomagnetic response as an output. This is an attractive feature of the ANNs which approves them to be suitable for geomagnetic activity forecasting.

Using the method of ANN, in Bochniček et al. (2007), Valach et al. (2007) and Valach et al. (2009), geoeffective solar processes have been specified and quantified on the basis of particular events as observed on the solar disc together with additional information concerning their properties and location on the solar disc. The magnetospheric response to the solar wind and the IMF conditions is complicated by "memory" effects, so each measurement inside the magnetosphere should be provided with information not only on the current state of the interplanetary medium, but also with data of the preceeding time interval, of an hour or more. We will attempt to contribute to this challenging topic in a forthcoming study with help of the recurrent neural networks.

\subsection{Measure of geomagnetic activity}

Geomagnetic activity is usually characterized by geomagnetic indices, the most common being the storm $D s t$ index and the planetary $K p$ index (Mayaud, 1980). Most indices have long records that allow statistical studies of the causes of activity and of related phenomena. Correlations between indices and possible drivers provide the basis for empirical prediction. The Dst index can serve as a good measure of the overall strength of the nearEarth electric currents, especially the ring current. It is obtained from selected geomagnetic observatories operating at equatorial regions. On the other hand, the global (planetary) $K p$ index is obtained as the mean value of the disturbance levels in the two horizontal field components, observed at 13 selected, subauroral observatories.

According to the series of geomagnetic indices $K p$ a four-grade scale was introduced in Bochniček et al. (2007) to evaluate the geomagnetic response. The particular degrees of the scale are: no response, weak, medium, and severe response. This scale is used for daily forecasts of the geomagnetic activity performed at the Regional Warning Centre Prague (http://www.ig.cas.cz/en/structure/observatories/ geomagneticobservatory-budkov/forecast-of-geomagnetic-activity/). Such measure of geomagnetic activity seems to be useful for the local implementation 
as a model output for mid-latitude regions. Moreover, it can serve to make the geomagnetic forecast accessible to broader scientific community and also to make it understandable to public.

\subsection{Towards the space weather forecast model}

Magnetospheric and solar wind observations, complemented by concurrent ground-based data, provide the basis for space weather modeling. The models can be designed to produce output in the form of geomagnetic indices and those can be compared with real records. It is important to realize that the ultimate goal of space weather research should be to forecast space weather and, at least, predict geomagnetic activity in terms of the geomagnetic index Dst as a function of time. Improved forecasting depends on new understanding through research, new advances in observation, and improved numerical and statistical models. But these activities alone will not improve forecasts unless there is significant effort made to transformation of these capabilities to space weather operations and to give forecasters the tools to synthesize the knowledge, observations and model output into a useful forecast.

\section{Mathematical model}

In what follows, we will demonstrate the capacity of empirical modeling focusing on a particular magnetic storm. In Romashets et al. (2008) a paraboloid 3D model of the magnetosphere has been used in order to compute magnetic fields in the region where the solar wind interacts with the Earth's magnetosphere. This part of the near Earth surroundings is known as the magnetopause and is usually of the main focus when modeling the interaction between the solar and planetary magnetic fields. The magnetic field exhibits a jump when moving from the solar wind (the external field) to the magnetosphere (the internal field). There is no normal component of the magnetic field at the magnetopause.

The geomagnetic activity measured in terms of the $D s t^{m}$ index can be related with the jump in magnetic field $\left[B_{t}\right]$ according to Romashets et al. (2008) as follows 
$D s t^{m}=\xi \sum_{k=0}^{N} \zeta^{k}\left[B_{t}\right]_{k}$.

The superscript ${ }^{m}$ stands for the model values. Here, $k$ denotes the contribution to the geomagnetic disturbance of flows that came in $k$ hours ago and $\xi$ is a free parameter to be determined later. The scaling parameter

$\zeta^{k}=\frac{1}{2}\left[1-\operatorname{sgn}\left(B_{z}\right)\right] \exp (-k / 12)$

was introduced to ensure that only the jump $\left[B_{t}\right]$ from a negative to a positive value be considered.

The magnitude of the jump $\left[B_{t}\right]$ across the magnetopause can be computed in a rather technical way and will not be reproduced here. Instead, we refer to the final expression for the $D s t^{m}$ index (formula (77) in Romashets et al. (2008)) which reads

$$
\begin{aligned}
D s t^{m} & =\xi \sum_{k=0}^{N} \zeta^{k}\left\{B_{z}\left[4.2629\left(\frac{V_{\infty}}{500}\right)\left(\frac{10^{6}}{T_{\infty}}\right)^{1 / 2}-1\right]-\right. \\
& \left.-34.2109\left(\frac{n_{\infty}}{5}\right)^{1 / 2}\left(\frac{V_{\infty}}{500}\right)\right\}_{k} .
\end{aligned}
$$

Here, the subscript $\infty$ stands for the undisturbed solar wind fields far before the interaction with the magnetosphere; $V_{\infty}$ is the velocity measured in $\mathrm{km} \mathrm{s}^{-1}, n_{\infty}$ is the particle density measured in $\mathrm{cm}^{-3}, T_{\infty}$ is the temperature measured in $\mathrm{K}, B_{z}$ represents the $z$ component of the IMF measured in $\mathrm{nT}$ and the model $D s t^{m}$ index is measured in $\mathrm{nT}$.

\section{Comparison with observations}

The record of 135 hours has been considered, commencing on August 1, 2010, to compute the model $D s t^{m}$ index for the magnetic storm of August 4, 2010. The entries in (3) are the hourly averaged solar wind data. They are provided by registration of the ACE satellite operating at the libration point L1. In Fig. 1, the IMF component $B_{z}$ is shown together with the magnetogram of the horizontal magnetic component $H$ registered at Hurbanovo 
observatory. The magnetic storm characteristic onset and return phase can be identified in magnetic field records.
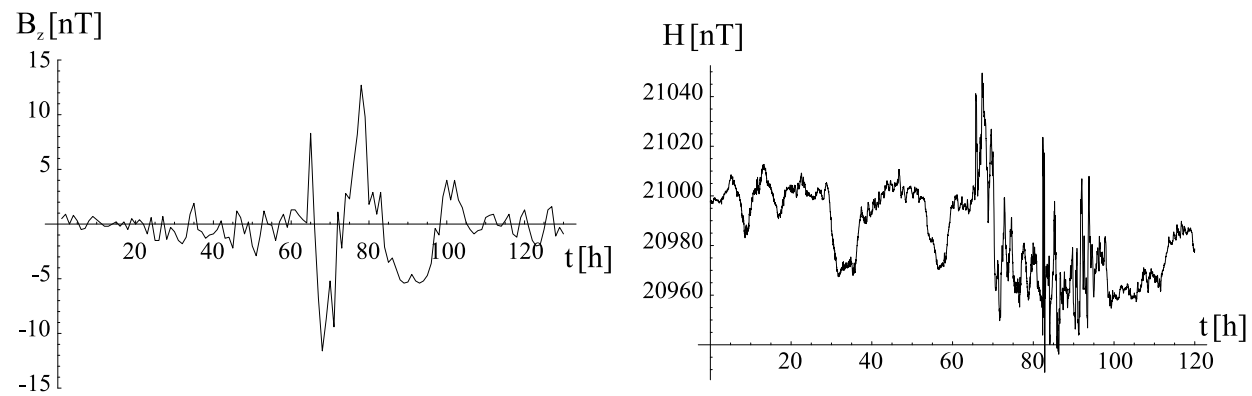

Fig. 1. The IMF component $B_{z}$ (left) registered by the ACE satellite and the magnetogram of the horizontal magnetic component $H$ (right) registered at Hurbanovo observatory.

In Eq. (3), the number of backward hourly steps $N$ has been left as a free parameter to be varied to ensure the best agreement with real data. The question is how much backward information about the solar wind properties is needed to achieve maximum similarity. The free multiplicator $\xi$ in Eq. (3) can be computed to minimize the normalized mean square error $N M S E$ defined by

$N M S E=\frac{1}{M^{2}} \sum_{i=1}^{M}\left[D s t_{i}^{m}-D s t_{i}\right]^{2}$,

where $M$ is the length of the record (in hours).

The parameter $N$ has been varied from $N=5$ to $N=45$. The real data $D s t$ together with the model values $D s t^{m}$ for different choices of $N$ are shown in plots in Fig. 2. The storm onset is better captured by the model for smaller $N$, though at the return phase better agreement can be seen for larger $N$. Thus, there can be some intermediate values of $N$ for optimum model output (see also Table 1). Varying $N$ is in contrast to Romashets et al. (2008) where the fixed value of $N=12$ has been used to model the extremely strong geomagnetic storm of July 14, 2000, known as the Bastille event. 

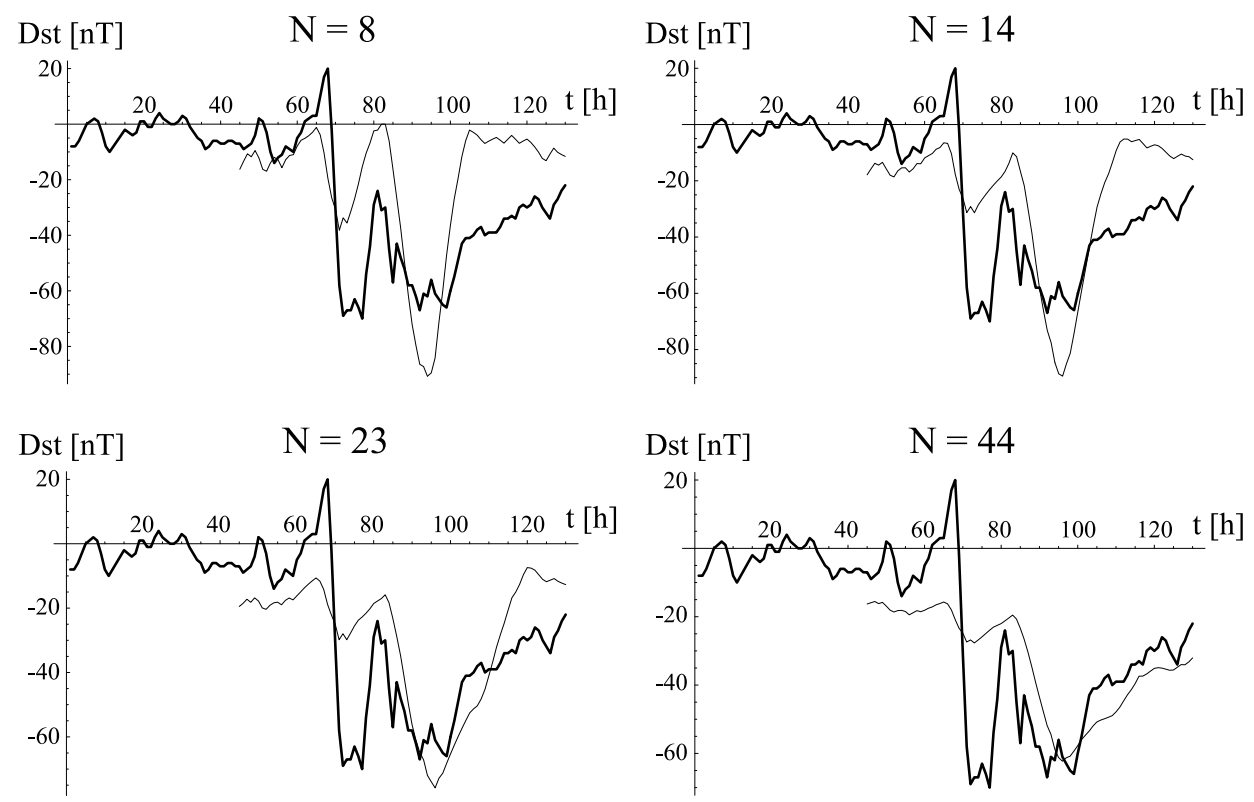

Fig. 2. The comparison of the real $D s t$ index (thick curve) and simulated $D s t^{m}$ index (thin curve) for various numbers of backward hourly steps, $N=8,14,23,44$.

Table 1. The values of the multiplicator $\xi$ and the normalized mean square error $N M S E$ for various backward hourly steps $N$

\begin{tabular}{|c|c|c|}
\hline $\mathrm{N}$ & $\xi$ & NMSE \\
\hline \hline 5 & 0.111 & 2.959 \\
\hline 8 & 0.085 & 2.678 \\
\hline 11 & 0.071 & 2.469 \\
\hline 14 & 0.061 & 2.314 \\
\hline 17 & 0.055 & 2.185 \\
\hline 20 & 0.049 & 2.091 \\
\hline 23 & 0.044 & 2.017 \\
\hline
\end{tabular}

\begin{tabular}{|c|c|c|}
\hline $\mathrm{N}$ & $\xi$ & NMSE \\
\hline \hline 26 & 0.041 & 1.974 \\
\hline 29 & 0.037 & 1.939 \\
\hline 32 & 0.035 & 1.907 \\
\hline 35 & 0.032 & 1.883 \\
\hline 38 & 0.030 & 1.887 \\
\hline 41 & 0.029 & 1.891 \\
\hline 44 & 0.027 & 1.896 \\
\hline
\end{tabular}

\section{Conclusions}

Synthetic record of the geomagnetic storm has been obtained using empirical model. It is observable that considering longer past data record (in terms 
of $N$ ) improves the model agreement with the return phase (see Fig. 2). The $N M S E$ decreases with $N$ and is lowest at $N=35$ (see Table 1) but in that case, the model lacks agreement with the storm onset. From the point of view of prediction, it would be desirable to capture the onset of the disturbance as well. Adjusting scalings and parameters in empirical expressions can be the possible way towards improving the model response. In a subsequent study, we expect to benefit from combining the empirical study together with the use of the method of ANN; thereby setting up a more comprehensive model. More extensive data sets will be analyzed for quiet solar wind conditions as well as for strongly disturbed solar wind conditions.

Acknowledgments. The support from the VEGA grants 2/0023/08, 2/0043/08 and $2 / 0059 / 08$ is acknowledged. The authors wish to thank the referee for valuable comments.

\section{References}

Bochníček J., Hejda P., Valach F., 2007: Solar energetic events in the years $1996-2004$. The analysis of their geoeffectiveness. Studia Geophys. Geod., 51, 439-447.

Gombosi T. I., DeZeeuw D. L., Groth C. P. T., Powell K. G., Clauer C. R., Song P., 2001: From Sun to Earth: Multiscale MHD Simulations of Space Weather, in Space Weather. Geophys. Monogr. Ser., 125, edited by P. Song, H. J. Singer, and G. Siscoe, 169-176, AGU, Washington, D. C.

Kim R.-S., Cho K.-S., Moon Y.-J., Kim Y.-H., Yi Y., Dryer M., Bong S.-C., Park Y.-D., 2005: Forecast evaluation of the coronal mass ejection (CME) geoeffectiveness using halo CMEs from 1997 to 2003. J. Geophys. Res., 110, A11104, doi:10.1029/2005JA011218.

Mayaud P. N., 1980: Derivation, Meaning, and Use of Geomagnetic Indices. Geophys. Monogr. Ser., 22, 154 p., AGU, Washington, D. C.

Robinson R. M., Behnke R. A., 2001: The U.S. National Space Weather Program: A Retrospective, in Space Weather. Geophys. Monogr. Ser., 125, edited by P. Song, H. J. Singer, and G. Siscoe, 1-0, AGU, Washington, D. C.

Romashets E. P., Poedts S., Vandas M., 2008: Modeling of the magnetic field in the magnetosheath region, J. Geophys. Res., 113, A02203, doi:10.1029/2006JA012072.

Valach F., Hejda P., Bochníček J., 2007: Geoeffectiveness of XRA events associated with RSP II and/or RSP IV estimated using the artificial neural network. Studia Geophys. Geod., 51, 551-562. 
Valach F., Revallo M., Bochníček J., Hejda P., 2009: Solar energetic particle flux enhancement as a predictor of geomagnetic activity in a neural network-based model. Space Weather, 7, S04004, doi:10.1029/2008SW000421. 